\title{
Influence of temperature and velocity on the emptying of silo hoppers
}

\author{
Marcus Ripp*, Robert Hesse and Sergiy Antonyuk \\ Institute of Particle Process Engineering, University of Kaiserslautern, Germany
}

\begin{abstract}
For the design of bulk material silos, a detailed knowledge of the flow properties of bulk materials is necessary. If the dynamic processes in a silo and, above all, the transition from a stationary bulk material to a flowing bulk material, the designer faces difficult challenges. In the following work a new measuring method is presented, with which it is possible to measure important information about the behavior of bulk goods in silos.
\end{abstract}

\section{Introduction}

The flow behaviour of bulk solids depends on a number of parameters. The flow parameters required for the design of silos are determined with shear cells at room temperature [1]. The results of the measurements with shear cells could use for the comparison of the flow properties of the bulk solids and for optimization of the silo discharge process. To predict the flow behavior in a silo it is necessary to know the flow property at the transition from passive to active dynamic stressing state or the transition of adhesion to sliding friction. Also the conditions of the slip-stick effect are important to know. Investigations show, that there is an influence of the velocity [1] and the temperature [2, 3] on the flow inside the bulk silo. These effects cannot be completely estimated in standard shear cells. Therefore, it cannot be assumed that there is always coulomb friction.

In the work a novel experimental setup will be presented which provides further details on the flow behavior of bulk materials.

\section{Measurement setup}

The newly developed experimental setup is shown in Fig. 1. A tube (diameter $100 \mathrm{~mm}$ ) was clamped in a tensile testing machine (universal Zwick/Roell testing machine, Fig. 2), and the tube was closed from below by means of a piston which can be displaced in the tube. Various bulk materials were charged into the tube $(240 \mathrm{~mm})$ through a recess in the upper part, which also functions as prevention for a potential vacuum during the shearing process. The bulk material was subsequently loaded with weights from above to simulate a higher filling level to obtain a uniform normal stress in the tube walls (Fig. 3). The tube was withdrawn from the bulk material column and the wall friction forces between the tube and filled bulk material were measured by force transducers.

During the removal of the tube with a defined velocity the particle-wall friction force and displacement are measured. To perform the measurements at different temperatures, the tube is covered with a heating jacket. The construction allows a variation of the diameter and the material of the tube.

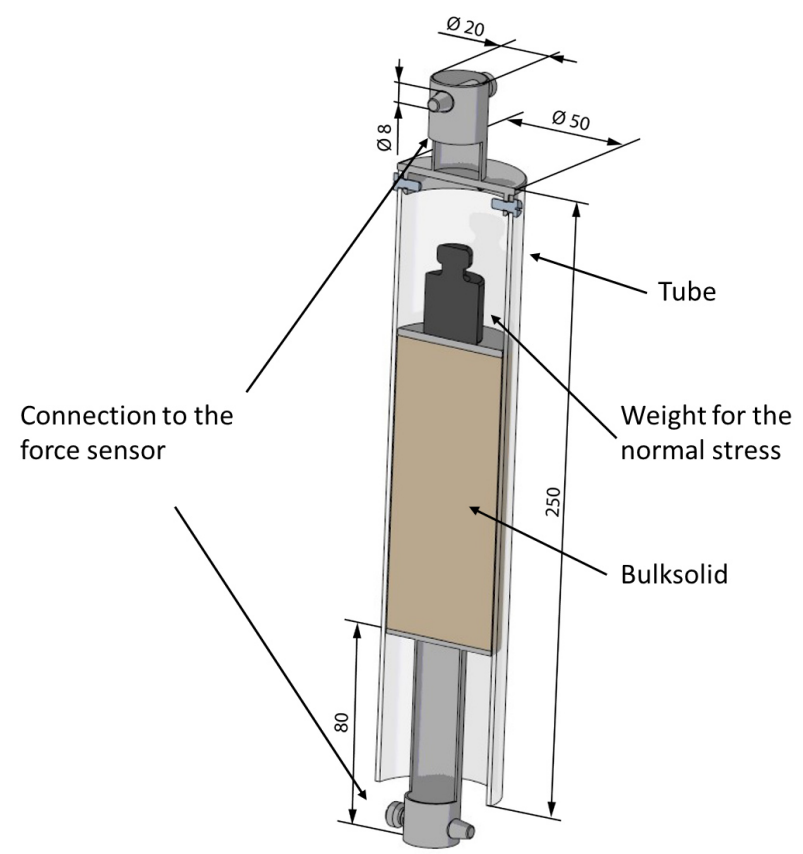

Fig. 1: Experimental setup for dynamic shear test with bulk solid (all dimensions in $\mathrm{mm}$ )

\footnotetext{
* Corresponding author: ripp@mv.uni-kl.de
} 


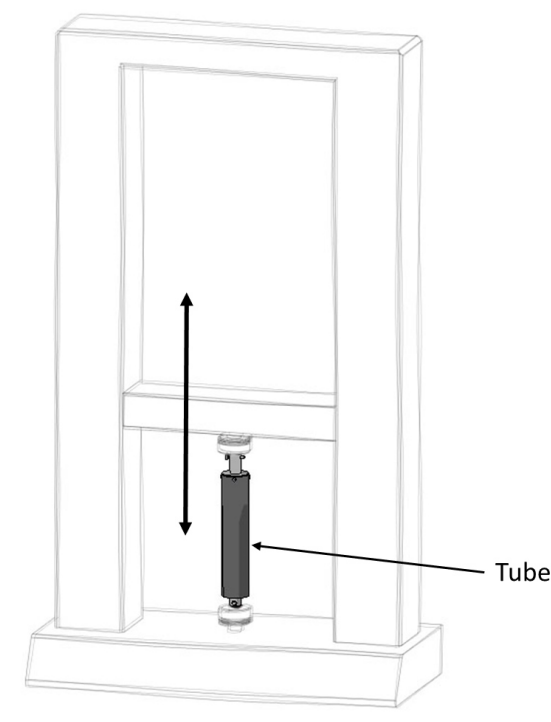

Fig. 2: Tension testing machine with the tube measuring cell

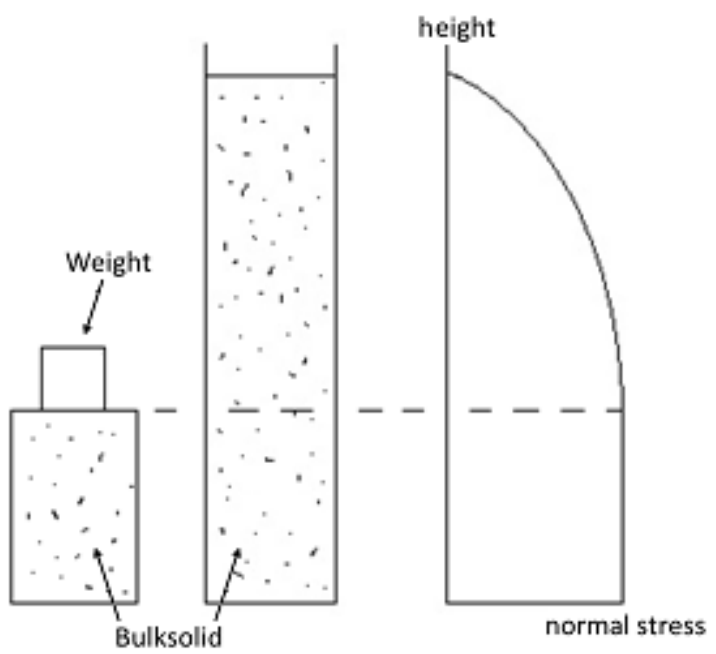

Fig. 3: Normal stress in the walls for varying bulk solid heights

Various plastic granules were used in the tests (Fig. 4 and 5).

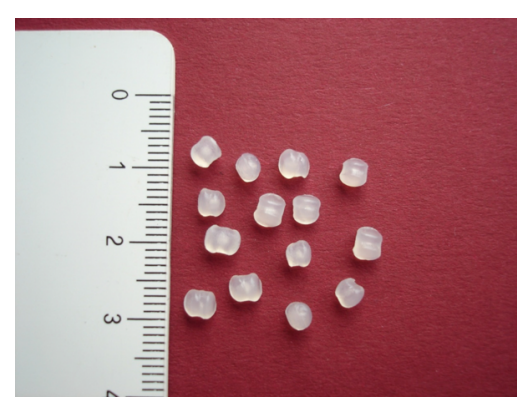

Fig. 4: Polyethylene Pellets (PE)

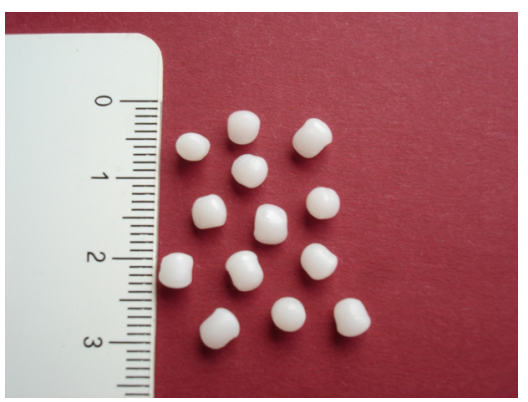

Fig. 5: Polypropylene Pellets (PP)

\section{Experimental results}

The results of the measurements with Polyethylene Pellets show very clearly that relatively large stickings have to be overcome during the start-up of the tube. A constant value for the sliding friction is established only after a travel distance of approximately $10 \mathrm{~mm}$ (Fig. 6). This sticking friction at the beginning of the discharge of a bulk silo could lead to problems during the operation of a bulk silo. In the further course of the measurement, a slip-stick effect can be recognized which is to be analysed in further experiments.

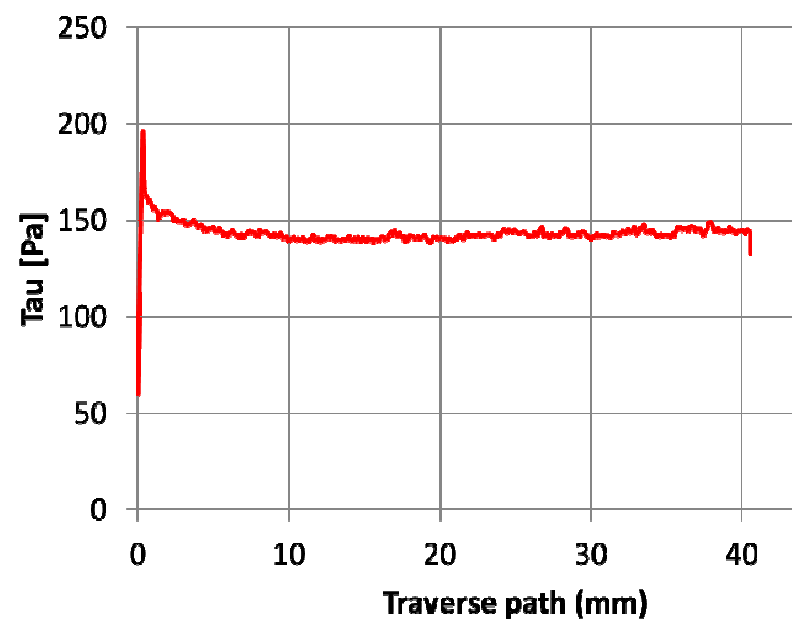

Fig. 6: measured wall shear stress (Tau) versus shear displacement for polyethylene granules at a constant velocity of $40 \mathrm{~mm} / \mathrm{min}$. (PE) 


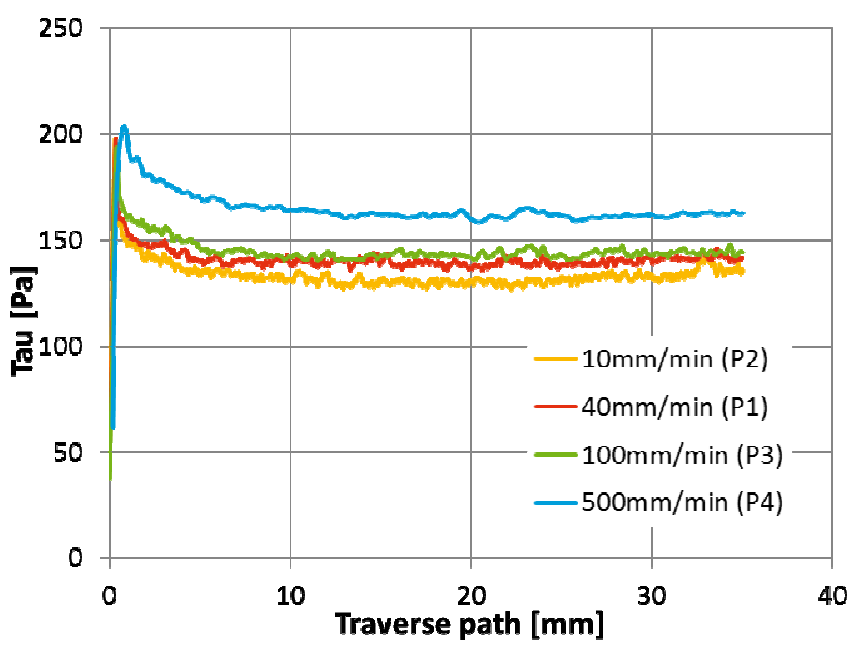

Fig. 7: Different drawing speeds of the tube with PE granules

Fig. 7 shows that different values for the sliding friction are set at different drawing speeds. This is a clear indication that there is no Coulomb friction in this case. If these results are transferred to tests in shear cells, the shear cell tests have to be adapted to the flow velocities in the silo-application.

In Fig. 8, results are shown in which the temperature of the measuring device was varied with a defined pull speed. Polypropylene (PP) was tested as granules.

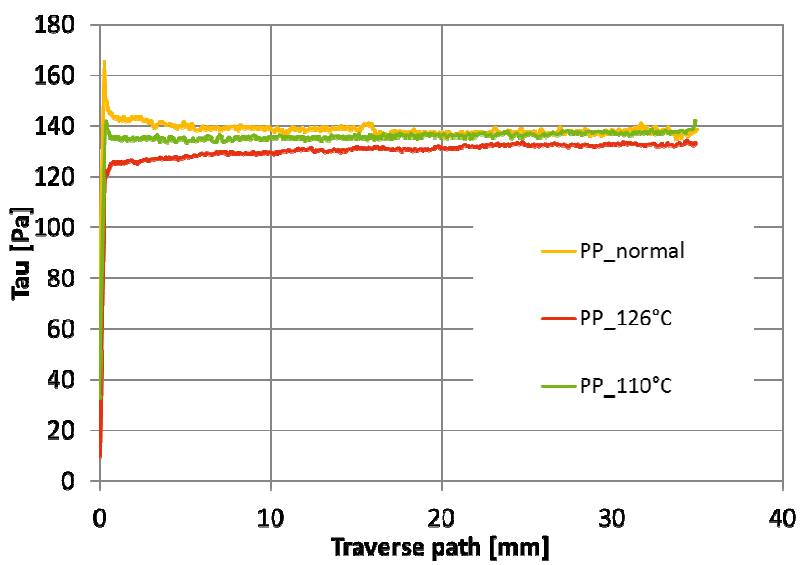

Fig. 8: Different temperatures of the tube with PP granules

The results show that the temperature has a significant influence on the wall shear stress in the pipe. Especially at the beginning of the measurement, a large difference of the measured results is to be determined depending on the temperature. The highest peak at the beginning of the movement of the tube was obtained for the material at the room temperature. With increasing the temperature, the effect of the sticking friction at the beginning of the movement is decreased. These results show very nicely that the wall friction depends on the surface properties of the particles. The surface of the particles becomes softer with increasing temperature, which leads to a reduction in the adhesion friction at the start of the measurement. Conversely, the stiffness of the plastic particles is responsible for the force at the start of the measurement. It is astonishing that, in the further course of the measurement, there is almost no difference in the sliding friction.

\section{Conclusion}

In bulk material technology, it is important to know the flow behavior of bulk solids very precisely. For many years, shear cell testers have been used, but they do not always give all the necessary information. In addition, it is often assumed that the friction between particles and particles and silo walls is a Coulomb friction. This is not always correct.

A new measuring system was developed at the Institute of Particle Process Engineering, University of Kaiserslautern, Germany, to provide more detailed information on the behavior of bulk solids in silos.

The measurements showed that a sharp increase in the shear stress can be observed with plastic granules at the beginning of the flow process. A slip-stick effect is also shown during the further course of the measurement. These measurements are a basis for further research, which will be supported by simulation methods in the future. It is planned that conclusions can be drawn about the actual processes in bulk material silos and thus the construction of bulk material silos can be made safer. This applies in particular to the dynamic behavior of bulk goods in silos and the transfer of vibrations to the silo construction.

In further experiments measurements were carried out at different temperatures with the new experimental apparatus. This showed a clear influence of the temperature on the flow behavior. These attempts to interpret the results of measurements at room temperature also clearly show that the influence of the temperature on the flow behavior of bulk materials is not always negligible.

\section{References}

1. D. Schulze, Powder and Bulk Solids, Behavior, Characterization, Storage and Flow (Springer Berlin Heidelberg New York, 2008)

2. M. Ripp, S. Ripperger, Chemical Engineering Science, 65, 13 (2010)

3. I. Tomasetta, D. Barletta, M. Poletto, Advanced Powder Technology, 24, 3 (2013) 